Jpn. J. Med. Mycol.

Vol. 39, 173-178, 1998

ISSN $0916-4804$

\title{
Short Report \\ Evaluation of Antifungal Activity of an Antifungal Drug by In Vitro Simulation of In Vivo Pharmacokinetics of the Drug against Fungal Hyphal Growth
}

\author{
Ki-Bong Oh ${ }^{1}$, Hideki Shirogane ${ }^{1}$, Hideaki Matsuoka ${ }^{1}$, Akira Niitsu ${ }^{2}$, \\ Satoru Yamada ${ }^{3}$, Hiroshi Kurata ${ }^{4}$, Mayumi Mochizuki ${ }^{5}$, Hikaru Kume ${ }^{6}$ \\ ${ }^{1}$ Department of Biotechnology, Faculty of Technology, Tokyo University of Agriculture and Technology, \\ 2-24-16, Nakamachi, Koganei, Tokyo 184-8588, Japan \\ ${ }^{2}$ Research and Development Division, Hidan Co., Ltd., \\ 627, Hananoi, Kashiwa, Chiba 277-0812, Japan \\ ${ }^{3}$ Research and Development Division, Bio-Giken Inc., \\ 1-5-8, Iwamoto-cho, Chiyoda-ku, Tokyo 101-0032, Japan \\ ${ }^{4}$ The Tokyo Kembikyoin Foundation, \\ 4-8-32, Kudanminami, Chiyoda-ku, Tokyo 102-0074, Japan \\ ${ }^{5}$ Department of Pathology, School of Medicine, Kitasato University, and \\ ${ }^{6}$ Department of Pharmacy, Kitasato University Hospital, \\ 1-15-1, Kitasato, Sagamihara, Kanagawa 288-0829, Japan \\ [Received : 27, November 1997. Accepted : 27, January 1998]
}

\begin{abstract}
An automatic drug concentration simulator (DCS) has been developed and its applicability has been demonstrated by in vitro simulation of the human plasma concentration-time curve of fluconazole (FLCZ) against hyphal growth of Candida albicans and Aspergillus fumigatus. The response of hyphal growth to FLCZ was continually monitored and analyzed using an automatic hyphal growth analyzing system (Bio-Cell Tracer). The simulated concentration of FLCZ by DCS was confirmed by HPLC. The DCS assay was reproducible with a mean coefficient of variation (C.V., $n=3$ ) of $5.38 \%$. When the growth of $C$. albicans hyphae was tested, there was a lag of onset of FLCZ effect between the time when FLCZ concentration became maximal ( $\mathrm{CMAX}, 7.95 \mu \mathrm{g} / \mathrm{m} l$ ) and the point at which hyphal growth ceased. In contrast, FLCZ was found inactive against $A$. fumigatus. The newly devised technique could provide clinicians with important information in determining optimal dosing regimens for antifungal drugs.
\end{abstract}

Key words : pharmacokinetics, fluconazole, in vitro simulation, hyphal growth rate, Candida albicans, Aspergillus fumigatus

\section{Introduction}

It is very clear that the serious nature of fungal infections has developed at an increasing rate over the last half century, coincidentally with the development of antibiotic, anti-inflammatory and immunosuppressive chemotherapies, and more recently with the emergence of AIDS ${ }^{1-3)}$. This increasing incidence of fungal infections is putting new demands on medical practice worldwide. There is only a limited range of antifungal drugs currently available and each of these has its

別刷請求先 : Hideaki Matsuoka

2-24-16, Nakamachi, Koganei, Tokyo 184-8588, Japan

Department of Biotechnology, Faculty of Technology,

Tokyo University of Agriculture and Technology shortcomings ${ }^{4)}$. The azole antifungal drugs are the most widely used antifungal drugs today, although resistance is emerging. For example, 5 years after the introduction of fluconazole, an orally active compound with excellent tissue penetration, an alarming proportion of Candida strains isolated from infected patients are resistant to it ${ }^{5)}$. There is a strong need for a new broad spectrum of fungicidal, nontoxic antifungal drugs, but unfortunately the development of new antifungal chemotherapies still lags behind that of antibacterial agents.

Recently, a system for the evaluation of dynamic growth of a single hypha (Bio-Cell Tracer, BCT) has been developed and its applicability to the assessment of the antifungal activity of antifungal 


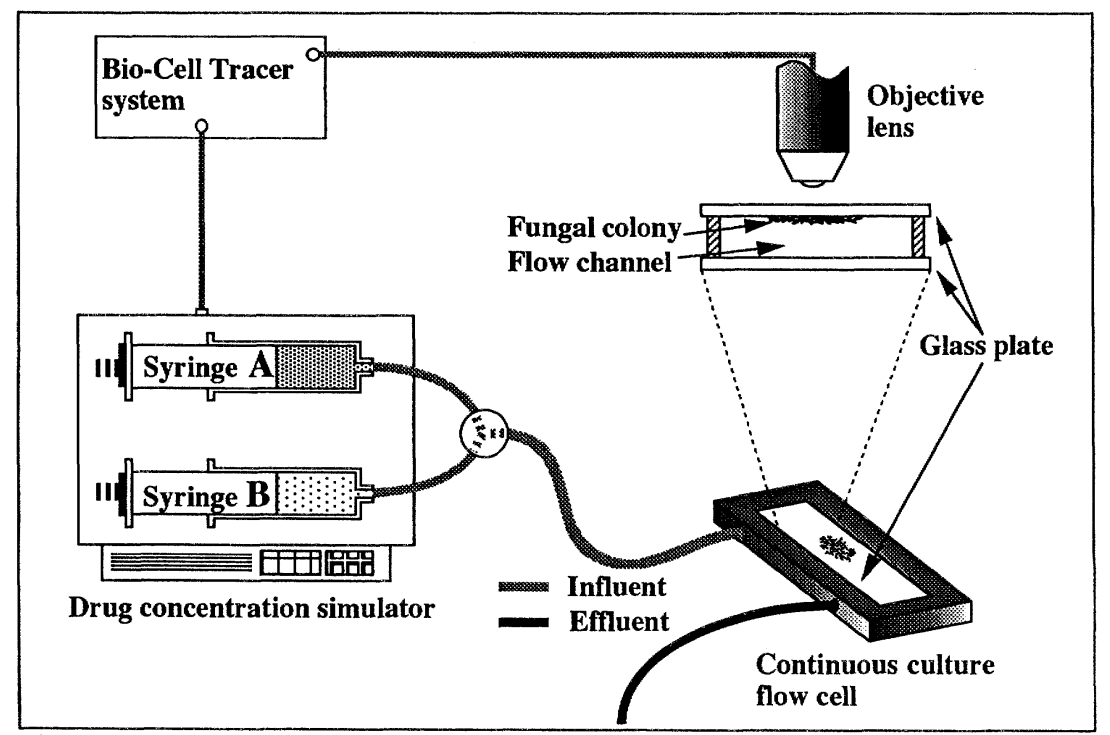

Fig. 1. Schematic representation of the automatic drug concentration simulator. Continuous culture-flow cell was used to monitor hyphal growth response in real time to antifungal drug. The flow cell is inoculated with fungi, which are allowed to attach to the inner surface of the glass plate, where they are visible under white-light illumination.

agents has been demonstrated ${ }^{6-8)}$. This system has enabled the determination of hyphal growth rate in real time in the presence or absence of various agents $^{9-11)}$. This microbioassay is based on the continuous measurement of the growth of a test hypha under a microscope. Such a continuous measurement of the same hypha is impossible by the conventional assay methods dealing with massive hyphae.

In the present study, we have developed an automatic system for the simulation of in vivo pharmacokinetics of an antifungal drug in vitro. The applicability of this system was demonstrated by in vitro simulation of the plasma concentrationtime curve of fluconazole against Aspergillus fumigatus and Candida albicans. The antifungal activities of fluconazole on the hyphal growth of test organisms were quantitatively evaluated using BCT.

\section{Materials and Methods}

\section{Organisms and antifungal drug}

Candida albicans KULM 83-0300 and Aspergillus fumigatus, Kuboyama strain, were tested. All antifungal experiments were conducted with yeast nitrogen base (Difco Laboratories) supplemented with $1 \%(\mathrm{w} / \mathrm{v})$ glucose (YNBG). The $\mathrm{pH}$ of the medium was adjusted to 7.0 with $\mathrm{K}_{2} \mathrm{HPO}_{4}$ ( $2.6 \mathrm{~g}$ per liter) and then sterilized by membrane filtration. Fluconazole (FLCZ, Diflucan intravenous solution, Pfizer Pharmaceuticals Inc., Tokyo, Japan) was diluted with YNBG to prepare the desired initial concentration.

\section{Automatic hyphal growth analyzing system (Bio-Cell Tracer)}

An automatic system, called the Bio-Cell Tracer (BCT), has been developed for the evaluation of antifungal activity on the basis of single hyphal growth rate in the presence or absence of various agents. The system construction and main function of $\mathrm{BCT}$ were described previously ${ }^{10)}$.

\section{Drug concentration simulator (DCS)}

The newly developed DCS system used in this study was a dual syringe infusion type, which was controlled by the microcomputer of BCT (Fig. 1). The initial values defined recording conditions such as the human plasma concentrationtime curve of FLCZ, syringe volume and diameter, medium flow rate, head volume of silicon tube, and initial antifungal drug concentration. According to those data, antifungal drug concentration in the continuous culture-flow cell was controlled automatically by adjusting the rate of pumping of dual syringes (syringes A and B in Fig. 1) as follows:

$$
\mathrm{V}_{1}=\mathrm{V}\left(\mathrm{P} / \mathrm{P}_{0}\right) \text { and } \mathrm{V}=\mathrm{V}_{1}+\mathrm{V}_{2} \text {, }
$$

where $\mathrm{P}$ : antifungal drug concentration in the continuous culture-flow cell; $\mathrm{P}_{0}$ : initial antifungal drug concentration in syringe A (YNBG plus antifungal drug); $\mathrm{V}$ : medium flow rate in the continuous culture-flow cell; $\mathrm{V}_{1}$ : the pumping rate of syringe $A ; V_{2}$ : the pumping rate of syringe $B$ (YNBG only). In this study, the medium flow rate in the continuous culture-flow cell was fixed at $5 \mathrm{ml} / \mathrm{h}$ and the initial concentration of FLCZ in the syringe A was $10 \mu \mathrm{g} / \mathrm{ml}$. 


\section{HPLC analysis}

The accuracy of DCS for the simulation of plasma concentration-time curve of FLCZ was evaluated by high performance liquid chromatography (HPLC). At each time point during the experimental period, $100 \mu l$ of liquid medium in the continuous culture-flow cell was sampled and the FLCZ concentration was analyzed by HPLC (Tosoh model SC-8020, Tosoh Corporation, Tokyo) equipped with a $\mu$-Bondapak $\mathrm{C}_{18}$ column ( $5 \mu \mathrm{m}, 15 \times 6 \mathrm{~mm}$ I.D., Waters Assoc., Milford, MA, USA ). Analysis of FLCZ was conducted with water-acetonitrile $(3: 1, \mathrm{v} / \mathrm{v})$ as the mobile phase at a flow rate of $1 \mathrm{ml} / \mathrm{min}$. FLCZ was detected by UV $A_{254}$ and the retention time was 2.6 min. A standard curve was constructed by plotting the area under the sample peak versus the FLCZ concentration. The FLCZ concentration in the continuous culture-flow cell during the test period was calculated by comparison with the standard curve.

\section{Antifungal activity assay}

A continuous culture-flow cell (Fig. 1) was used to monitor hyphal growth response during the experimental period. The inner surface of a glass plate was coated with poly-L-lysine (poly-Llysine hydrobromide $m w$ 70,000 150,000, Sigma) to which yeast cells and hyphae adhered. This enabled a continuous observation of hyphal growth without focus adjustment during the test period. Coating was done by pouring a $0.01 \%(\mathrm{w} / \mathrm{v})$ solution of poly-L-lysine onto the glass plates and incubating for $15 \mathrm{~min}$ at $25^{\circ} \mathrm{C}$, then removing the solution and allowing the plates to dry in a sterileair-flow cabinet under UV irradiation.

C. albicans was preincubated in $\mathrm{YNBG}$ at $25^{\circ} \mathrm{C}$ on a laboratory shaker at $120 \mathrm{rpm}$. After $24 \mathrm{~h}$, the yeast cells were harvested and resuspended in sterile distilled water at a concentration of $10^{6}$ cells $/ \mathrm{m} l$. Ten $\mu l$ of this suspension was placed on the poly-L-lysine coated glass plates. The yeast cells were left for $20 \mathrm{~min}$ to settle and excess, nonadherent cells were washed off using YNBG and then set in the flow cell. The flow cell was mounted on the microscope stage of the BCT and connected to the DCS. YNBG in syringe B (Fig. 1) was then introduced into the flow cell at 5 $\mathrm{m} l / \mathrm{h}$. The temperature was constantly controlled at $37^{\circ} \mathrm{C}$. In the case of $\mathrm{A}$. fumigatus, the flow cell was prepared in the same manner as described above. Conidia were allowed to germinate at 37 ${ }^{\circ} \mathrm{C}$ for $20 \mathrm{~h}$ under a constant flow rate prior to use.

After confirming that the hyphal growth rate of $C$. albicans or $A$. fumigatus was constant for

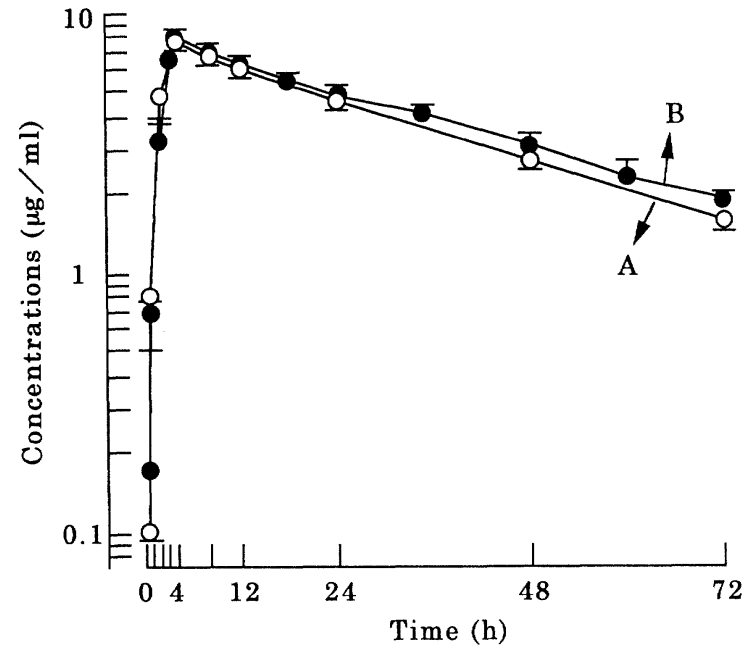

Fig. 2. Comparison of plasma concentration-time curve of fluconazole (A) and simulated curve of fluconazole in the continuous-culture flow cell by the automatic drug concentration simulator based on curve A (B). A: plasma concentration-time curve of fluconazole in healthy male volunteers $(n=9)$ after a single oral administration of $400 \mathrm{mg}$ using a $100 \mathrm{mg}$ capsule (TMAX, $3.6 \pm 0.3 \mathrm{~h}$; $\left.\mathrm{C}_{\operatorname{MAX}}, 7.95 \pm 0.29 \mu \mathrm{g} / \mathrm{ml} ; \mathrm{T}_{1 / 2}, 28.9 \pm 1.3 \mathrm{~h}\right) . \mathrm{B}$ : at each point, fluconazole concentration in the flow cell was sampled and analyzed by HPLC as described in the text. The data are expressed as mean $\pm \mathrm{SD}$ for three separate experiments.

$4 \mathrm{~h}$, the simulation experiment was started by introducing the YNBG containing FLCZ in syringe A. According to the plasma concentration-time curve, the drug concentration in the flow cell was controlled by DCS. At the same time, the growth rate of test hyphae was traced automatically using BCT.

\section{Results}

Under the chromatographic conditions described above, the standard curve for FLCZ at the concentration range studied was linear. The HPLC assay showed an excellent correlation between the area under the sample peak and the standard drug concentrations $(0 \sim 10 \mu \mathrm{g} / \mathrm{ml})$, with $\mathrm{r}$ of $>0.99$ and a sensitivity of $0.1 \mu \mathrm{g} / \mathrm{m} l$. The FLCZ concentrations of serial samplings in the flow cell were calculated by comparison with the standard curve.

In order to demonstrate the accuracy and reproducibility of the DCS system, the plasma concentration-time curve of FLCZ (line A in Fig. 2) was fed into the microcomputer of BCT. The medium flow rate in the flow cell was fixed at $5 \mathrm{ml} / \mathrm{h}$ and the initial concentration of FLCZ in syringe A (Fig. 1) was $10 \mu \mathrm{g} / \mathrm{ml}$. According to the plasma concentration-time curve data of FLCZ, a set of dual syringes of DCS was controlled by the microcomputer of BCT. At each time point, 100 
$3-\mathrm{A}$

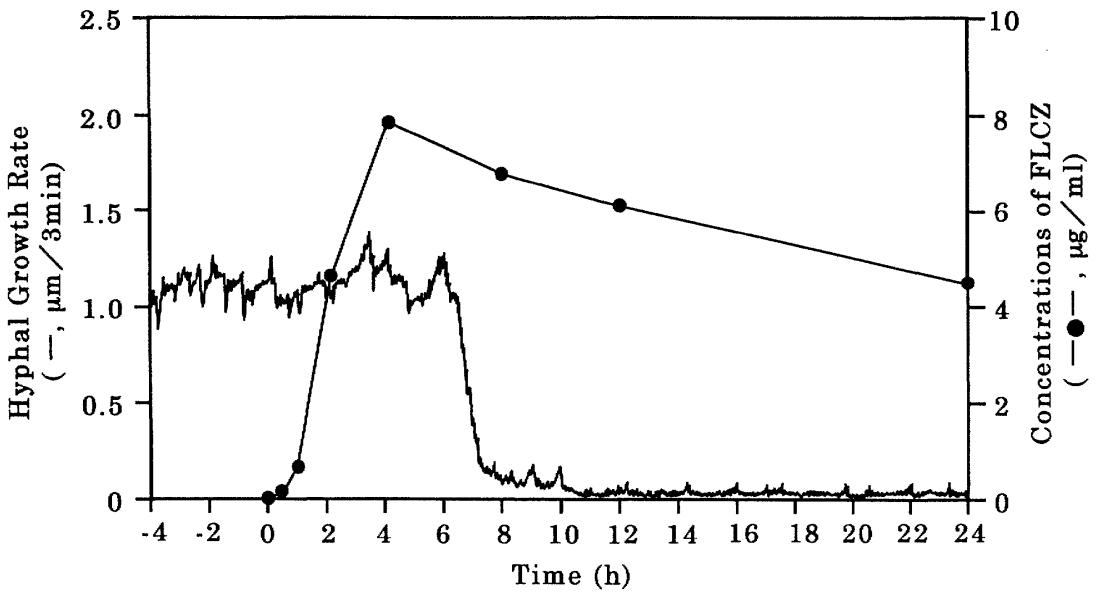

$3-\mathbf{B}$

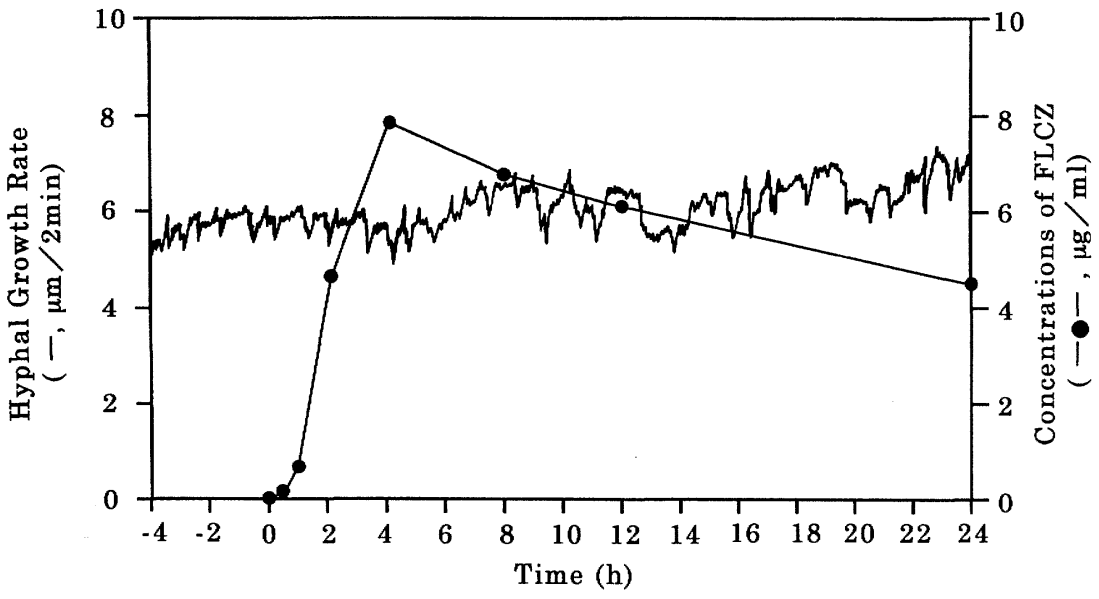

Fig. 3. Antifungal activity of fluconazole (FLCZ) against hyphal growth of C. albicans (A) and A. fumigatus (B). Plasma concentration-time curve of FLCZ was simulated using the drug concentration simulator. At the same time, hyphal growth rate of test fungus was analyzed by BCT system. Experiments were done in triplicate for each strain. Each growth curve shows a representative result from the average data obtained with 5 test hyphae. Data varied by less than $10 \%$.

$\mu l$ of liquid medium in the flow cell was sampled and analyzed by HPLC. The simulated curve of FLCZ in the DCS system is depicted in Fig. 2 (line B). The variability of DCS method as determined by coefficients of variation (C.V.) in repeated analyses of aliquots of the sample during the experimental period was $8.30 \%$ for the lowest concentration assayed and was $2.14 \%$ for the highest concentration assayed, with a mean of $5.38 \%$. These data indicate that the DCS method is reproducible within the same assay run.

Microscope examination revealed that yeast and hyphal forms of the C. albicans strain cultured in YNBG medium at $25^{\circ} \mathrm{C}$ for $24 \mathrm{~h}$ comprised approximately $98 \%$ and $2 \%$, respectively. After $2 \mathrm{~h}$ at $37^{\circ} \mathrm{C}$, however, about $95 \%$ of the inoculum yeast cells transformed into hyphae. The germ tubes elongated to form complete hyphae, and the growth rate reached a steady state for approx. $2 \mathrm{~h}$. To confirm the constant level of hyphal growth rate in this study, the growth rate of C. albicans hyphae was estimated for $4 \mathrm{~h}$ before simulating the pharmacokinetics of FLCZ in plasma. The average growth rate of hyphae during this period was estimated at $0.33 \pm 0.03 \mu \mathrm{m} / \mathrm{min}$ (mean $\pm \mathrm{SD}, \mathrm{n}=20$ ). In $A$. fumigatus, the average and standard deviation of the hyphal growth rate were determined as $2.83 \pm 0.32 \mu \mathrm{m} / \mathrm{min} \quad(\mathrm{n}=22)$.

The plasma concentration-time curve of FLCZ was simulated by using DCS against hyphal growth of $C$. albicans and A. fumigatus (Fig. 3 ). The average hyphal growth response curve of $C$. albicans to FLCZ is illustrated in Fig. $3 \mathrm{~A}$. There was a delay in onset of FLCZ effect between the time FLCZ concentration became maximal and the point at which hyphal growth ceased. The growth rate of test hyphae was stable and no appreciable inhibitory effect was observed until the FLCZ concentration reached the maximum concentration $\left(\mathrm{C}_{\mathrm{MAX}}, 7.95 \pm 0.29 \mu \mathrm{g} / \mathrm{ml} ; \mathrm{T}_{\mathrm{MAX}}\right.$, 
$3.6 \pm 0.3 \mathrm{~h}$ ). After $2.5 \mathrm{~h}$, however, the growth rate of test hyphae decreased slowly and stopped completely after an additional $4.5 \mathrm{~h}$. In contrast, FLCZ was found inactive against $A$. fumigatus (Fig. $3 \mathrm{~B}$ ).

\section{Discussion}

This study analyzes for the first time the effect of antifungal drug by in vitro simulation of the in vivo pharmacokinetics of the drug against fungal hyphal growth. The simulated concentration of FLCZ by DCS method on the basis of the plasma concentration-time curve was reproducible with a mean C.V. of $5.38 \%$ (Fig. 2). The response of fungal hyphae to an antifungal drug can be continually monitored and analyzed in real time as demonstrated with FLCZ.

Activity tests in a laboratory with antifungal drugs are designed either to determine the minimum amount of drug needed to inhibit the growth of a particular organism in vitro, or to measure drug concentrations in persons receiving antifungal treatment. In most standard in vitro susceptibility tests, however, antifungal activity determinations are made following continual exposure of the organism to a particular drug for a period of $24 \mathrm{~h}$ or longer 12-15). Numerous factors can affect the results of tests with these drugs, and MICs often correlate poorly with the clinical results of antifungal treatment ${ }^{16-18)}$. In contrast, in the in vivo situation organisms are usually exposed to a drug of which concentration is fluctuating and not constant ${ }^{19-22)}$. There can be little doubt that the most useful method for assessment of the activity of an antifungal drug is to test it in vivo. However, animal models are time-consuming, laborious and expensive test methods in practice, so their use is largely restricted to development and assessment of new antifungal drugs. To determine the effective dosage regimen of an antifungal drug, the in vitro monitoring of the pharmacokinetics of the drug in blood against clinical isolates seems to be one of the best indices for determining its pharmacological effects.

In the present study with FLCZ, a delay in the onset of the drug effect on the hyphal growth of C. albicans was observed (Fig. $3 \mathrm{~A}$ ). The inhibitory effect of FLCZ was delayed approximately $2.5 \mathrm{~h}$ after the FLCZ concentration reached the maximum concentration. Galgiani and Stevens ${ }^{23)}$ noted that there was a "time to drug effect" for miconazole with approximately equalled 1.5 doublings of yeasts when tests were begun with stationary phase starting inocula. However, this was inapparent with organisms introduced to drug in logarithmic phase. A subsequent report by McIntyre and Galgiani ${ }^{24)}$ found a similar delay in onset of FLCZ effect (approximately $3 \mathrm{~h}$ ), but introducing FLCZ during logarithmic phase did not seem to eliminate the delay. The measurements in our present report elegantly corroborate this effect. The reason for the delay in onset of the FLCZ effect is unknown. However, this delay suggests effects on cell metabolism requiring processing time, rather than a more direct action on the cell membrane, which would have more immediate impact.

The newly devised technique of DCS could provide clinicians with important information in determining optimal dosing regimens for antifungal drugs. The limitations of the present study include the limited number of strains studied as well as the technical sophistication for clinical application. Our efforts are now directed towards the resolution of these technical problems.

\section{References}

1) Morrison VA, Haake RJ, Weisdorf DJ: NonCandida fungal infections after bone marrow transplantation: risk factors and outcome. Am J Med 96 : 497-503, 1994.

2) Dupont B, Denning DW, Marriott D, Sugar A, Viviani MA, Sirisanthana T: Mycoses in AIDS patients. J Med Vet Mycol 32 (Suppl. 1) :6577, 1994.

3) Armstrong D: Treatment of opportunistic fungal infections. Clin Infect Dis 16:1-9, 1993.

4) Lyman CA, Walsh TJ : Systemically administered antifungal agents. A review of their clinical pharmacology and therapeutic applications. Drug 44: 9-35, 1992.

5) Van den Bossche H, Marichal P, Odds FC: Molecular mechanisms of drug resistance in fungi. Trends Microbiol 2:393-400, 1994.

6) Matsuoka H, Ii Y, Takekawa Y, Teraoka T: Evaluation of antifungal volatile compounds on the basis of the elongation rate of a single hypha. Appl Environ Microbiol 56 : 3779-3784, 1990.

7) Yamada S, Cao J, Sumita O, Kurasawa K, Kurata H, Oh KB, Matsuoka H: Automatic antifungal activity analyzing system on the basis of dynamic growth process of a single hypha. Mycopathologia 118:65-69, 1992.

8) $\mathrm{Oh} \mathrm{KB}$, Matsuoka H, Sumita O, Takatori K, Kurata $\mathrm{H}$ : Evaluation of antifungal activity of antimycotics by automatic analyzing system. Mycopathologia 118: 71-81, 1992.

9) Oh KB, Matsuoka H, Nemoto Y, Sumita O, Takatori K, Kurata H: Determination of antiAspergillus activity of antifungal agents based on the dynamic growth rate of a single hypha. Appl Microbiol Biotechnol 39:363-367, 1993.

10) Oh KB, Yang HC, Matsuoka H, Yamamoto A, Kurata $\mathrm{H}$ : Combined effect of amphotericin B and flucytosine on hyphal growth rate of Candida 
albicans estimated at a single hypha level. J Med Vet Mycol 33 : 191-195, 1995.

11) Taguchi H, Miyaji $M, X u$ M, Nishimura $K$, Yoshida $\mathrm{T}$ : Studies on an antifungal effect of miconazole on Aspergillus fumigatus by Bio-Cell Tracer. Jpn J Med Mycol 36:33-37, 1995.

12) Espinel-Ingroff A, Kish CWJ, Kerkering TM, Fromtling RA, Bartizal KF, Galgiani JN, Villareal K, Pfaller MA, Gerarden T, Rinaldi MG, Fothergill A : Collaborative comparison of broth macrodilution and microdilution antifungal susceptibility tests. J Clin Microbiol $30: 3138-3145$, 1992.

13) Fromtling RA, Galgiani JN, Pfaller MA, EspinelIngroff A, Bartizal KF, Bartlett MS, Body BA, Frey C, Hall G, Roberts GD, Nolte FB, Odds FC, Rinaldi MG, Sugar AM, Villareal K : Multicenter evaluation of a broth macrodilution antifungal susceptibility test for yeasts. Antimicrob Agents Chemother 37:39-45, 1993.

14) Martin E, Maier F, Bhakdi S: Antagonistic effects of fluconazole and 5-fluorocytosine on candidacidal action of amphotericin B in human serum. Antimicrob Agents Chemother 38 : 1331 1338, 1994.

15) National Committee for Clinical Laboratory Standards: Reference method for broth dilution antifungal susceptibility testing for yeasts; Proposed standard. NCCLS Document M27-9, Vol. 12, No.25, 1992.

16) Drutz DJ : In vitro antifungal susceptibility testing and measurement of levels of antifungal agents in body fluids. Rev Infect Dis 9:392-397, 1987.
17) Fromtling RA: Overview of medically important antifungal azole derivatives. Clin Microbiol Rev 1:187-217, 1988.

18) Sara LR: Candida and Aspergillus infections in immunocompromised patients: An overview. Rev Infect Dis 13:487-492, 1991.

19) Benson JM, Nahata MC: Drug review. Clinical use of systemic antifungal agents. Clin Pharm 7 : 424-438, 1988.

20) Brammer KW, Farrow PR, Faulkner JK : Clinical pharmacology. Pharmacokinetics and tissue penetration of fluconazole in humans. Rev Infect Dis 12 (Suppl. 3) : S318-S326, 1990.

21) British Society for Antimicrobial Chemotherapy Working Party: Laboratory monitoring of antifungal chemotherapy. The Lancet 337 : 1577-1580, 1991.

22) La Delfa I, Zhu QM, Mo Z, Blaschke TF : Fluconazole is a potent inhibitor of antipurine metabolism in vivo in mice. Drug metab Disp $17: 49-53,1989$.

23) Galgiani JN, Stevens DA : Turbidimetric studies of growth inhibition of yeasts with three drugs: Inquiry into inoculum-dependent susceptibility testing, time of drug effect, and implications for current and newer methods. Antimicrob Agents Chemother 13 : 249-254, 1978.

24) McIntyre KA, Galgiani JN : In vitro susceptibilities of yeasts to a new antifungal triazole, $\mathrm{SCH}$ 39304: Effect of test conditions and relation to in vivo efficacy. Antimicrob Agents Chemother 33 : 1095-1100, 1989. 